\title{
Art et Nature, quelques remarques sur la poétique de Shakespeare
}

Art and Nature: a few remarks on Shakespeare's poetics

\section{Henri Suhamy}

\section{(2) OpenEdition}

\section{Journals}

\section{Édition électronique}

URL : http://journals.openedition.org/shakespeare/1043

DOI : 10.4000/shakespeare.1043

ISSN : 2271-6424

Éditeur

Société Française Shakespeare

\section{Édition imprimée}

Date de publication : 1 novembre 2007

Pagination : 131-145

ISBN : 2-9521475-3-1

\section{Référence électronique}

Henri Suhamy, "Art et Nature, quelques remarques sur la poétique de Shakespeare ", Actes des

congrès de la Société française Shakespeare [En ligne], 24 | 2007, mis en ligne le 30 mars 2010, consulté le 06 mai 2019. URL : http://journals.openedition.org/shakespeare/1043 ; DOI : 10.4000/

shakespeare. 1043

Ce document a été généré automatiquement le 6 mai 2019.

(c) SFS 


\title{
Art et Nature, quelques remarques sur la poétique de Shakespeare
}

\author{
Art and Nature: a few remarks on Shakespeare's poetics
}

Henri Suhamy

1 Dans le prolongement du vaste essai de Victor Hugo, auteur en 1864 d'un éloge de Shakespeare considéré comme le poète par excellence, parler de Shakespeare poète peut susciter des envolées mystiques - romantiques dans un premier temps, puis enrichies de considérations plus modernes, empruntées à diverses disciplines. Sur ce dernier point il n'est pas difficile de démontrer que l'œuvre de Shakespeare illustre ce que Jakobson appelle la fonction poétique du langage, et toutes les autres, y compris la fonction métalinguistique. Bien entendu le qualificatif de poète appliqué à notre auteur ne se réfère pas seulement aux poèmes narratifs et lyriques qui représentent une partie importante de sa production, près de six mille vers. Il faut, pour prendre toute la mesure de cette notion, aborder directement le théâtre comme foyer de poésie. Or, selon une conception implicite et d'autant plus difficile à extirper de l'esprit du public, il pourrait exister une opposition entre la mimésis théâtrale et la poésie, s'ajoutant à un préjugé qui fait du théâtre un art mineur, ayant besoin d'un supplément d'inspiration et de stylisation pour accéder à la véritable dignité poétique. Cette dignité étant atteinte, la notion de théâtre poétique évoque des œuvres enrobées d'une sorte de halo, imprégnées d'une catharsis qui n'attend pas le dénouement de la pièce pour se manifester, mais qui fait des acteurs les célébrants d'un rituel collectif dès les premières paroles qu'ils s'adressent les uns aux autres mais en direction du public, habitées par un sens profond et général, d'où émane une transcendance qui plane au-dessus de la linéarité terre-à-terre du scénario. En plus du contenu supposé visionnaire du texte théâtral, hors du temps et de l'espace quotidiens, du symbolisme des images, de la lumière unifiante d'une Weltanschauung, des références aux grands mythes fondateurs, jugés capables de provoquer un sentiment d'évasion ou d'élévation, il ne faut pas négliger dans cette perspective la forme versifiée que prend ce texte le plus souvent - l'ensemble de l'œuvre dramatique de Shakespeare contient environ 75000 vers - et qui, quel que soit son contenu, et bien que cette forme ne se réduise pas à un uniforme conventionnel, manifeste, même en l'absence des balises 
acoustiques que constituent les rimes, son appartenance à un langage autre que celui de la communication ordinaire. Le metteur en scène Jack Gold me disait un jour que quand on dirige des acteurs dans Shakespeare il faut s'efforcer de les ramener à terre, car, sans aller jusqu'à leur faire rejoindre les mythiques sphères dont la musique provoque l'extase de Pericles, le rythme, la mélodie du vers, le découpage du discours en segments qui donne au corps même de l'acteur l'habitude de respirer en mesure, ainsi que l'alchimie du langage, les poussent à s'envoler vers une stratosphère dorée où s'estompent le caractère distinctif des personnages ainsi que la tension des conflits. Il y aurait une étude à mener sur les influences respectives de la prose et du vers dans la caractérisation des personnages. Ceux qui s'expriment beaucoup en prose, comme Falstaff, Ford, Benedick ou Hamlet apparaissent comme ayant des traits plus marqués, plus idiosyncrasiques que ceux qui ne parlent qu'en vers. J'ai lu récemment, mais je ne me rappelle plus où, qu'à la fin de sa longue vie John Gielgud disait qu'il regrettait d'avoir au cours de sa carrière, et surtout dans Shakespeare, atténué le réalisme dramatique au profit d'une déclamation quasi musicale, qui tendait à embellir et adoucir le texte théatral, à désindividualiser les personnages. Il regrettait, en somme, d'avoir sacrifié la nature à l'art. Ces considérations ont préoccupé Shakespeare lui-même, qui avait des choses à dire sur la poétique, tout en les exprimant de façon oblique et prismatique, à travers ses personnages. Certaines enjolivures provoquent parfois des réserves de la part du public, des critiques, et des producteurs de spectacles, prompts à accuser l'écriture poétique de s'étouffer à force de s'étoffer, de se figer en poétisme. Par exemple les paroles prononcées dans Macbeth par l'un des meurtriers de Banquo, attendant au crépuscule l'arrivée de sa victime :

The west yet glimmers with some streaks of day.

Now spurs the lated traveller apace,

To gain the timely inn; and near approaches

The subject of our watch. (3.4.5-8)

ont été sévèrement jugées. "The lovely lines ... are not gutter-bred » écrit brutalement Harley Granville-Barker, cité par Kenneth Muir, lequel cite aussi, pour faire contrepoids, un commentaire d'un certain Bethell, justifiant la présence de cette poésie décorative et périphrastique dans la bouche d'un personnage de qui on ne l'attend pas, et au cœur d'un passage où devrait régner une grande tension dramatique : « it is ... dangerous to speak of certain characters as being more 'poetic' than others: in poetic drama everyone necessarily speaks poetry ${ }^{1}$. " Mais rien n'est jamais univoque dans Shakespeare et l'on pourrait aussi sentir dans le passage cité un décalage voulu entre le style et les circonstances, une certaine dose de parodie et d'humour noir, comme il s'en trouve beaucoup dans Macbeth. T.S. Eliot occupe une position intermédiaire dans le débat lorsqu'il dit que ce genre d'écriture appartient typiquement à la période juvénile de Shakespeare. Commentant le passage de Hamlet où Horatio décrit en termes anthropomorphiques l'arrivée du matin,

But look, the morn in russet mantle clad,

Walks o'er the dew of yon high eastern hill. (1.1.171-2)

Eliot dit que ces vers pourraient être tirés de Romeo and Juliet et ne sont plus vraiment à leur place dans Hamlet ${ }^{2}$, comme si de tels excès de poétisation trahissaient un manque de maturité. Pourtant de telles envolées sont fréquentes dans Shakespeare. En l'occurrence les deux passages cités traduisent avec joliesse un certain émerveillement, prolongé par une tendance à la mythification, devant deux phénomènes très naturels et très quotidiens, le soir et le matin, ce qui illustre une conception traditionnelle et populaire de la poésie, selon laquelle sa fonction première est de nous faire redécouvrir le monde, et la 
vie, et la nature, avec une fraîcheur enfantine, tous les matins, et tous les soirs aussi. Sur ce point il faut reconnaitre que la poésie de Shakespeare offre une profusion presque égale à celle de la nature, mais c'est bien ce qu'on lui reproche parfois, d'où l'application d'un remède efficace, le sécateur. Depuis le dix-septième siècle on assiste régulièrement à des représentations où la luxuriance du langage poétique de Shakespeare subit un élagage aussi impitoyable que celui que le jardinier de Richard II inflige aux abricotiers. Pourtant notre auteur savait certainement ce qu'il faisait. Comme rappelé plus haut, la poétique n'est pas une discipline réservée aux commentateurs universitaires et autres inspecteurs des travaux finis. Elle constitue d'abord un recueil de règles, de méthodes, à la disposition des créateurs eux-mêmes, puisque comme chacun sait, le mot grec poiètès signifie créateur, voire faiseur ou fabricant. Comme le remarque avec fierté sir Philip Sidney dans sa Défense de la poésie, le mot maker a circulé comme synonyme de poet. Incidemment, et contrairement à Shelley plus tard dans sa propre Défense de la poésie, Sidney, qui d'ailleurs consacre une grande partie de son traité à l'art dramatique, se méfie de l'assimilation entre poète et prophète. Composer une pièce de théâtre, la mener jusqu'à son dénouement tout en accrochant l'intérêt et l'émotion du public, c'est un travail de poète au sens étymologique. De plus Shakespeare ne cesse d'inclure dans ses pièces et dans ses poèmes des réflexions sur la création artistique en général, peinture, sculpture, musique, architecture, mais aussi sur les arts mineurs tels que la joaillerie. C'est l'imitation ou plutôt la recréation de la nature qu'il semble admirer le plus dans les arts représentatifs, comme on le voit par exemple dans la longue description du tableau imaginaire et idéal représentant la chute de Troie dans The Rape of Lucrece, alors que la bizarrerie et l'ingéniosité retiennent son attention dans le domaine des arts décoratifs. Le mot art (au sens d'art, bien sûr, et de technique ou de savoir-faire, en dehors de la deuxième personne $\mathrm{du}$ verbe to be, qui donne lieu cependant à un jeu de mots dans le sonnet 75 , «thou art all my art ») revient soixante-dix fois, souvent opposé proverbialement à la nature, et, ce qui nous confronte à une interrogation inquiétante, souvent aussi accompagné d'une nuance défavorable, au sens d'artifice trompeur, de dissimulation hypocrite, de maquillage aguicheur ou de sorcellerie pernicieuse. On ne rencontre pas dans Shakespeare l'adjectif artistic, ni le mot artifice, mais artificial est utilisé sept fois, doté d'une nuance péjorative. La contestation la plus radicale s'exprime par la voix d'Apemantus qui, à Timon lui demandant au sujet d'un tableau :

Wrought he not well that painted it? répond:

He wrought better that made the painter, and yet he's but a filthy piece of work. (1.1.197-9)

L'art et la nature pourraient constituer les deux pôles d'une dissertation à la française, menée selon une dialectique à l'allemande : thèse - antithèse - synthèse. On commence par opposer l'art à la nature, puis on les réunit, et, comme à la fin d'un congrès politique, on termine par une motion de synthèse, habilement équilibrée, conciliant des notions antagonistes tout en admettant leurs différences. Cette gymnastique argumentative n'aurait pas surpris Shakespeare, puisqu'il la pratique lui-même, comme dans le débat sur les OGM entre Perdita et Polixenes. Il n'en reste pas moins que le plus souvent l'art et la poésie apparaissent comme étant opposés à la nature et à la sincérité. Bien entendu il faut relativiser ces jugements puisque aucun des personnages de Shakespeare, même Hamlet, ne peut passer pour son porte-parole. Les condamnations de l'art ou de la poésie, la vieille accusation selon laquelle les poètes sont des menteurs, associant l'art en général à une 
perception déformée du réel ou à la séduction frauduleuse, placées dans la bouche de ceux qui représentent un pouvoir hiérarchique plus ou moins oppresseur peuvent, par un processus analogue à l'ironie dramatique, se retourner contre ceux qui les prononcent. Quand John of Gaunt dénonce l'effet pernicieux de la poésie sur les chefs d'État, ou que Hotspur clame que la cultiver n'est pas digne d'un gentilhomme, on soupçonne que de telles opinions n'engagent que ceux qui les expriment. Dans le Sonnet 66 (« Tired with all these, for restful death I cry»), la censure contre l'art figure parmi les calamités publiques : "And art made tongue-tied by authority ». On peut cependant se demander si Shakespeare n'a pas parfois douté de la raison d'être de son art, bien que ce type d'interrogation puisse paraître démodé, surtout si on lui attribue un présupposé vaguement biographique. Depuis qu'un certain consensus règne au sujet de The Tempest, considérée comme une parabole sur la colonisation, et non comme le produit d'une réflexion sur l'art, sur ses illusions, sa possible inutilité, ou au contraire sur sa puissance, son utilité profonde, mais quelque peu ésotérique et provoquant la méfiance aussi bien des titulaires du pouvoir politique que de la foule, il est sans doute incorrect de voir en Prospero une projection au moins partielle de l'auteur à la veille ou l'avant-veille de son retour à Stratford et de son abandon de la poésie. Pourtant le thème pathétique du désenchantement de l'enchanteur ne manque pas de pertinence quand on essaie de s'interroger sur le rôle que tient la poésie dans la vie d'un poète. De même Orlando déclare qu'il ne supporte plus de jouer la comédie, et de substituer la vie intérieure à la vie tout court :

I can live no longer by thinking. (As You Like It, 5.2.50)

6 Ce thème apparaît de diverses façons bien avant The Tempest. On trouve dans Shakespeare une vingtaine d'occurrences du mot poésie, sous les deux formes anglaises de poetry et poesy, ainsi qu'une vingtaine du mot poet. Ces références à la poésie et au métier de poète ont, comme on l'a vu, presque toujours des connotations moqueuses et péjoratives, mais, comme annoncé, on finit par des synthèses fondées sur l'ironie et l'ambiguïté, retournant contre leurs auteurs certains sarcasmes ou réflexions sceptiques. L'exemple le plus connu se trouve dans A Midsummer Night's Dream, quand, au cours d'une digression apparente qui porte peut-être sur le sujet principal de la pièce, Thésée dénonce la poésie comme le produit fumeux d'une cervelle en feu et en dérangement. Mais il en arrive à exprimer le contraire de ce qu'il veut démontrer, et fait du poète à la fois un créateur et un messager entre la terre et le ciel :

The poet's eye, in a fine frenzy rolling,

Doth glance from heaven to earth, from earth to heaven,

And as imagination bodies forth

The forms of things unknown, the poet's pen

Turns them to shapes and gives to airy nothing

A local habitation and a name. (5.1.12-7)

D'autres occurrences métapoétiques ou métastylistiques rejoignent ce qui a été dit au sujet de la poétique en tant que métier, métier d'art, et de la versification. On y perçoit la même dualité, de l'humour, mais aussi le reflet de préoccupations sérieuses. Le mot rime a surtout pour fonction de former un couple antithétique avec reason, rappelant l'antinomie proverbiale qui oppose la poésie à la rationalité. Rime, de même que numbers, constituent surtout des métonymies ou synecdoques, renvoyant à la poésie versifiée, mais le mot numbers se réfère aussi au compte des syllabes, ce que confirme l'allusion faite par Quince aux ballades en six et huit ${ }^{3}$. Le mot pentameter, utilisé abondamment de nos jours, ne figure pas dans le vocabulaire de Shakespeare. Au-delà des considérations techniques, 
la place qu'occupe la poésie dans la société donne lieu à des notations plus ou moins obliques et railleuses. Les critiques et professeurs de poésie, tel Holofernes dans Love's Labour's Lost, n'y échappent pas; dans le passage amusant où ce maître d'école épluche avec sévérité le sonnet qu'il a intercepté et donne des leçons de creative writing à Nathaniel, se trouve à la fois une satire du pédantisme et un reflet des préoccupations artisanales de l'auteur. On note aussi, en lisant Shakespeare, qu'en son temps la poésie figurait déjà dans les programmes scolaires - mais peut-être faudrait-il en 2006 dire figurait encore plutôt que déjà - où elle était censée contribuer à l'éducation des jeunes personnes. Dans The Taming of the Shrew, Gremio déclare qu'il a trouvé pour Bianca un jeune précepteur qui fera l'affaire :

... for learning and behaviour

Fit for her turn, well-read in poetry,

And other books, good ones. (1.2.167-9)

8 L'apprentissage de la poésie accompagne l'acquisition des bonnes manières. Plus troublante est la présence de poètes professionnels sur la scène shakespearienne. Que Cinna le poète se fasse massacrer par la foule, non seulement parce qu'il porte le nom d'un conspirateur, anecdote saumâtre tirée de Plutarque, mais parce qu'il a écrit de mauvais vers, détail ajouté par Shakespeare, ou bien que dans la même pièce le poète qui intervient inopportunément pendant la dispute des deux chefs républicains apparaisse comme un bouffon inepte, ne doit sans doute pas être interprété comme révélant un goût morbide pour l'autodérision, bien qu'on puisse trouver étrange que dans Julius Caesar les seuls passages un peu comiques consistent à faire de l'humour, et de l'humour noir, aux dépens des poètes. Dans Timon of Athens, le personnage qui ne porte pas de nom, mais qui est nommé Poète dans les didascalies, représentant une corporation, comme le peintre et le joaillier, donne de la sienne une image plus inquiétante. Il fait peu modestement l'éloge de son inspiration. La poésie coule de lui naturellement dit-il. Pourtant l'allégorie dont il donne le résumé relève de la tradition, tout en fournissant un prologue à la tragédie, car comme toujours, Shakespeare montre simultanément plusieurs facettes des thèmes qu'il traite, que ce soit la politique, la guerre, l'amour, la sauvagerie et la civilisation, ici, la poésie. D'autre part ce poète joue un rôle de flatteur, de parasite, sa verve prétendument intarissable et spontanée se met au service des riches et des puissants. Il se révèle à la fin de la pièce comme un filou. Certains commentateurs ont peut-être été tentés de voir dans Timon of Athens une pièce à clés, et de chercher un nom précis à donner à ce poète anonyme. Cela paraît peu probable. Cette satire des gens de lettres, qui fait partie des traditions littéraires, constitue peut-être une sorte de vaccination contre les doutes qui assaillent de temps en temps les membres de cette profession, ou certains d'entre eux, et pourquoi pas, le plus illustre d'entre eux. C'est pourquoi la part d'autocritique, ou du moins d'examen de conscience que contient l'étrange portrait du poète que propose Shakespeare dans Timon of Athens mérite quelque considération. Le rôle social du poète est remis en cause, comme on l'a vu, d'une manière quasi moderne. La transformation au cours des âges du barde traditionnel, chantre et mémorialiste d'une tribu tout entière, en poète de cour, âpre au gain, au service d'une oligarchie ou d'une bourgeoisie, incite à la réflexion. L'auteur de Timon a pu éprouver quelque honte en se souvenant des dédicaces qu'il adressait au comte de Southampton une quinzaine d'années auparavant. La publicité que fait le poète de lui-même en se présentant comme un vaticinateur inspiré, ou, anticipant sur La Fontaine - comme un produit de la nature produisant à son tour des poèmes à la manière d'un arbre, réconciliant ainsi art et nature, avait peut-être de quoi irriter l'artisan que Shakespeare revendiquait d'être. Dans la dédicace de The Rape of 
Lucrece Shakespeare parle avec modestie, fausse modestie sans doute, comme le veut le rituel en pareil cas, de ses "untutored lines ». Que la modestie soit sincère ou feinte, l'expression contient l'idée qu'un vrai poète ne produit pas ses vers spontanément, mais qu'il a besoin d'abord de suivre un apprentissage sous la direction d'un maitre. Cette prise de distance à l'égard des pompes et des œuvres de la poésie officielle ou à la mode du temps occupe presque tout l'espace dans Love's Labour's Lost et prend la forme de la parodie. Mais il s'agit d'une parodie subtile, élégante, en partie complice des pratiques et jeux de langage qu'elle tourne gentiment en dérision. On n'imagine pas Shakespeare composant une farce intitulée Les précieuses ridicules - en l'occurrence ce serait plutôt Les précieux ridicules, car dans Love's Labour's Lost, le bons sens prosaïque appartient aux femmes. Il se sentait certainement trop solidaire de ses confrères poètes, professionnels ou amateurs, pour lancer contre eux des charges ravageuses.

9 À ceux qui reprochent à l'art de trop s'écarter de la nature, les partisans de l'art pour l'art ont parfois répondu par des paradoxes provocateurs, tel Oscar Wilde clamant que l'art n'a pas à imiter la nature, mais que de son côté c'est la nature qui imite l'art. De tels propos font sourire et sont considérés comme de simples boutades, lancées à l'appui de l'esthétisme doctrinal. Pourtant on peut tirer du texte de Shakespeare des pensées analogues. Ainsi dans Titus Andronicus le viol de Lavinia et l'endroit où ce crime a été perpétré sont douloureusement remémorés à la lumière d'une lecture d'Ovide. Titus s'exclame alors:

Ay, such a place there is, where we did hunt,-

0 , had we never, never hunted there,-

Pattern'd by that the poet here describes,

By nature made for murthers and for rapes. (III.i.55-8)

10 À quoi Marcus répond, en écho:

0 , why should nature build so foul a den

Unless the gods delight in tragedies. (59-60)

11 Ainsi en l'espace de six vers, nous avons une référence livresque à Ovide; apparaissent le mot poet, le verbe describes, et, plus frappants encore, le participe pattern'd, le verbe build, et le mot tragedies. La nature, nommée elle aussi, à la fois architecte et bâtisseuse, dessine et construit des théâtres de plein air où se déroulent des tragédies. De son côté le récit d'Ovide semble avoir servi de modèle ou de mode d'emploi aux barbares qui ont violé et mutilé Lavinia. On finit par ne plus savoir si c'est la nature qui imite l'art ou l'art la nature, tant leurs activités se ressemblent et se suivent. Toute l'œuvre de Shakespeare contient des ambivalences et références de ce genre, qu'il serait imprudent de réduire au rang de métaphores et autres figures de style. Sur bien des sujets le premier commentateur de Shakespeare est Shakespeare lui-même, et pour le comprendre il n'est pas toujours nécessaire de décortiquer le texte à l'aide d'outils anachroniques.

12 Au sujet de l'éternel débat sur les relations entre l'art et la nature, il faut rappeler que le langage est naturel à l'homme, alors qu'il n'existe pas dans la nature à l'état brut, physique ou biologique. Les structures du langage l'apparentent à l'art, et sa fonction poétique, pour reprendre l'expression jakobsonienne, lui est inhérente. D'autre part, parmi les quelque deux cents références métapoétiques qu'on rencontre dans l'œuvre de Shakespeare, l'art poétique, au sens professionnel, figure abondamment, avec des mots comme sonnets, ballads, verse, line, metre, numbers, déjà cité. Le mot rhythm est absent du vocabulaire shakespearien, de même que stress, mais on y trouve accent et cadence, une seule fois il est vrai. Holofernes parle pompeusement de « the golden cadence of poesy » ( 
Love's Labour's Lost, IV.ii.118). Il mentionne même la matérialisation graphique des élisions sous la forme des apostrophes (115). Quand l'auteur des Sonnets se réfère à une technique qui requiert plus de savoir-faire que d'inspiration, il montre une certaine modestie, à l'opposé des vantardises du poète de Timon of Athens. Il laisse entendre que l'art du vers requiert un soin minutieux; de plus il s'écarte de certaines traditions idéalistes, pétrarquistes et autres qui accompagnent souvent le genre, comme dans les fameux sonnets 129 et 130, « Th'expense of spirit in a waste of shame », et " My mistress' eyes are nothing like the sun ». Son lyrisme, certes profond et intense, apparait maîtrisé, intellectualisé, annonçant aussi bien la poésie du $\mathrm{XvII}^{\mathrm{e}}$ siècle appelée péjorativement «métaphysique » par Dryden, qui voulait dire en fait philosophique ou cérébrale, que le classicisme de Dryden lui-même ; la démarche qui conduit au distique final, généralement aphoristique, adopte souvent l'allure d'un raisonnement, ou du moins d'une rhétorique argumentative.

13 Le mot rhétorique vient d'être prononcé, non sans quelque préméditation. Si l'on parle de rhétorique en partant d'une idée exaltée de la poésie, on a l'impression de baisser d'un cran. Mais en empruntant le chemin inverse, si à partir du degré zéro de l'écriture on s'élève progressivement vers l'écriture poétique, on admettra que la rhétorique constitue un premier ou un second degré, une étape intermédiaire. Or au théâtre la rhétorique est présente partout, et elle montre au commentateur un double visage, une double origine. Elle émane de l'auteur, évidemment, mais des personnages aussi, en vertu de l'illusion mimétique. La notion de rhétorique est d'abord à prendre au premier sens, d'art de la persuasion, avant d'en arriver au sens dérivé, qui concerne les divers procédés de stylisation littéraire. Sur la scène, les personnages ne cessent d'agir les uns sur les autres, et quelquefois sur eux-mêmes, par la parole. Persuasion, séduction, menace, insultes, défis, exhortation, apitoiement, plaidoyer, accusation, toutes les postures, tous les procédés rhétoriques se trouvent dans Shakespeare. Même la guerre, notamment après les invectives homériques que s'adressent les adversaires, apparait comme la continuation de la rhétorique par d'autres moyens. Mais la rhétorique constitue une étape vers la poésie, du moins dans le cadre du présent exposé. Comment passe-t-on de la fonction conative du langage à la fonction poétique ? Ces deux fonctions cohabitent déjà au sein de la rhétorique, qui se définit à la fois par l'intention persuasive du locuteur, et par le façonnage du discours selon des formes plus ou moins conventionnelles ou inventives. Elles s'associent et parfois se dissocient, se défont l'une l'autre, comme cela arrive au malheureux Polonius quand il s'empêtre dans ses procédés oratoires, grâce auxquels il voulait donner de la force et du piquant à ses paroles. La reine l'interrompt sans ménagement et lui ordonne de mettre un peu moins d'art dans ses discours, car l'art, encore lui, assimilé à un maniérisme artificieux, complaisamment introverti, nuit non seulement à l'expression de la vérité, mais aussi à l'intelligibilité. Pour passer au registre supérieur, celui de la poésie, sous sa forme la plus extérieurement perceptible et conventionnelle, à savoir la versification imposée au discours, qu'il soit narratif, lyrique ou dramatique, on constate que les commentaires, d'ailleurs assez rares qu'elle suscite, vont dans deux directions opposées. Comme dans le cas de la rhétorique, selon leur sensibilité et leur doctrine certains commentateurs pensent que l'usage du vers intensifie l'expression, tandis que d'autres estiment qu'il tend à uniformiser et atténuer les caractères et les passions. Il y a aussi ceux qui pensent, sans forcément le dire, que l'écriture versifiée n'est, surtout au théâtre, qu'une sorte de péplum linguistique, produisant schématisation, monotonie et grandiloquence. On retrouve ici ce qui a été 
rappelé dès le commencement, tout art tend vers une certaine abstraction, un certain éloignement de la réalité immédiate. Il semble donc raisonnable de pencher vers la deuxième théorie, tout en se réservant le droit d'examiner les cas où le vers se met au service de l'expression. Sur ce point j'aborderai rapidement un aspect technique de la question. Le rythme poétique en anglais est fondé sur l'exploitation périodique des accents. Il existe deux types d'accents, qui se subdivisent eux-mêmes en deux autres catégories. Premièrement les accents lexicaux, inhérents à la langue anglaise, qui se divisent en accents principaux et accents secondaires. Dans un mot comme controversy, il y a un accent principal sur la première syllabe, un accent secondaire sur la troisième. L'un et l'autre procurent au rythme les accents ou ictus dont il a besoin pour animer le vers. L'évolution de la langue fait que certains accents secondaires paraissent aujourd'hui plus conventionnels que linguistiquement naturels. Par exemple la désinence finale dans le célèbre vers de Henry $v$ :

But we in it shall be remembered, (4.3.59)

Deuxièmement les accents non lexicaux, à savoir les accents conventionnels et les accents intonatifs. L'ictus conventionnel tombe sur un monosyllabe grammatical qui pourrait ne pas être accentué. Par exemple dans le premier vers du premier sonnet :

From fairest creatures we desire increase

Le pronom we, se trouvant en sixième position, et encadré par deux syllabes manifestement atones, ce qui constitue d'ailleurs une marque de fabrique du vers shakespearien, peut fournir au vers l'accent dont il a besoin pour maintenir le rythme en mouvement. En fait le récitant dispose d'une certaine latitude sur ce point. Le fait de devoir non seulement prononcer la désinence finale mais en plus l'accentuer paraît artificiel et archaïque à certains usagers. Last but not least l'accent intonatif. Il concerne surtout les monosyllabes, mais alors que l'accent conventionnel implique la soumission de la parole à une forme préétablie, l'accent intonatif permet aux énonciateurs d'exprimer des intentions particulières, tout en contribuant au rythme poétique. Dans ce vers du Sonnet 18 :

But thy eternal summer shall not fade,

16 l'emphase sur le pronom thy personnalise le propos tout en se trouvant à sa place dans la séquence iambique du vers. On peut en dire autant à propos des pronoms we et it dans le vers de Henry v cité précédemment : "But we in it shall be remembered». Le rythme se plie à l'expression personnelle, et inversement celle-ci épouse le rythme. L'intonation ne concerne pas seulement les ictus rythmiques. Elle imprime ses courbes à la mélodie du langage et laisse une large place à l'interprétation individuelle. Quant au mètre, au sens propre, il a pour fonction de calibrer la phrase sur un modèle répétitif et préétabli, mais les décalages, les accidents de plus en plus nombreux qui se produisent contribuent à l'expressivité du discours. Le vers représente donc un cadre bien dessiné, mais à l'intérieur duquel les acteurs peuvent se sentir à l'aise pour déployer leur personnalité et leur conception des rôles qu'ils incarnent.

17 Ces quelques remarques techniques avaient pour but de relancer une question déjà posée à propos de la rhétorique, qui peut sembler naïve : dans une œuvre théâtrale écrite en vers, qui s'exprime en vers, l'auteur ou les personnages? Le bon sens incite à répondre que c'est évidemment l'auteur, d'abord parce que c'est lui qui a écrit la pièce, qui l'a versifiée avec précisément l'intention de transfigurer l'anecdote dramatique et les créatures fictives qui y figurent. On peut dire qu'en plus de l'auteur c'est l'environnement culturel où il vit et travaille qui impose cette mise en forme, que certains jugent 
archaïque. Un universitaire américain du nom de Paul Fussell ${ }^{4}$ a même soutenu la thèse que la versification classique avait une fonction politique, parce que en obligeant les poètes à se soumettre à des règles, on leur inculquait la discipline et l'obéissance, attitudes que sans doute ils transmettaient à leurs lecteurs et auditeurs par imprégnation. Cette théorie n'est pas très sérieuse, mais elle exprime à sa façon l'idée, rarement exprimée à haute voix mais présente dans beaucoup d'esprits, qui voit dans la métrique traditionnelle un carcan dont la poésie moderne a bien fait de se passer. Il est vrai aussi que beaucoup de gens pensent que ce type de commentaire ne peut pas s'appliquer à Shakespeare, lequel passe parfois pour avoir pratiqué une métrique très souple, voire irrégulière ; mais cette opinion se fonde sur certaines apparences plus que sur un examen attentif du texte. Il ne faut pas confondre la souplesse et la ductilité avec le laisser-aller. Pour revenir à la question posée, est-ce l'auteur ou les personnages qui parlent en vers, ainsi qu'en prose, peut-être serait-il fructueux, à titre d'hypothèse de travail, de suspendre l'incrédulité et d'imaginer que ce sont les personnages qui sont doués d'élocution poétique. Tout d'abord la prosodie shakespearienne n'est pas uniforme. Elle varie à l'intérieur d'une même œuvre, elle évolue dans le temps, allant des formes carrées et martelées du début au style plus fluide et plus soluble dans l'air de la fin, grâce à l'abondance des terminaisons féminines, des enjambements, des ambiguïtés rythmiques. Elle laisse une grande place à l'intonation individuelle, permettant aux acteurs de participer au travail poétique. Elle laisse à la prose une place variable, mais non négligeable, puisque environ 27 pour cent du texte est écrit en prose. D'où un effet de modulation reflétant les situations dramatiques, le milieu social, les caractères, les attitudes, qui va de la prose au vers non rimé, et de celui-ci aux vers rimés, lesquels offrent plusieurs types de combinaisons: rimes plates, rimes croisées, séquences rappelant les sonnets, doggerel dans quelques comédies, jusqu'aux insertions de vers lyriques, de poèmes et chansons présentés comme tels, de citations ou d'extraits de théâtre dans le théâtre. Ces vers non dramatiques, au nombre de 2000 environ, attribués à l'invention des personnages, constituent une mise en abyme, parfois parodique et burlesque, parfois complaisamment virtuose, de la poésie à l'intérieur d'un texte luimême poétique. Cet effet prend parfois un aspect plus subtil. Par exemple au cours du dialogue entre Helena et le roi de France, au deuxième acte de All's Well That Ends Well, passant du blank verse au vers rimé, l'un et l'autre paraissent visités par une inspiration venue d'ailleurs. On sent l'intervention de l'auteur, s'efforçant de nous convaincre qu'il existe une transcendance au-dessus de la quotidienneté de la vie. Quand on revient au décasyllabe non rimé, le plus fréquent puisqu'il occupe environ $66 \%$ du total, on peut avoir l'impression, par contraste avec les formes plus ostensibles de la stylisation poétique, que, au moins pendant que dure l'illusion théâtrale, il appartient aux personnages eux-mêmes, puisque le plus souvent il se prête à l'expression du moment, tout en musicalisant le langage ordinaire. On peut dire de Shakespeare que son style atteint le summum de l'art, que la construction de ses pièces, même les plus foisonnantes, semble obéir docilement à la nature, quoi qu'en ait dit Tolstoï, et que cette sorte de Sprechgesang que constitue chez lui le vers non rimé opère une fusion entre ces deux entités contradictoires. L'art théâtral tend son miroir à la nature, dit Hamlet, mais il y a peut-être un art dans la nature, pour reprendre le mot de Polixenes, comme il y a de la poésie dans le langage, le saint langage, honneur des hommes. Le manifeste de Lautréamont selon lequel la poésie doit être faite par tous, non par une étroite corporation, trouve peut-être un semblant d'application dans l'existence du théâtre poétique. Les personnages de Shakespeare sont de beaux parleurs, même les pires 
moralement, et sous l'habillage formel, leurs propos font presque toujours affleurer un fonds commun de sensibilité, de préoccupation spirituelle, d'appréhension esthétique de l'univers. En déléguant ses pouvoirs aux personnages qu'il crée, et auxquels acteurs et spectateurs tendent à s'identifier, le théâtre, par extension et par procuration, fait participer chacun de nous à l'expression et à la vision poétiques.

\section{NOTES}

1. Édition Arden de 1951, p. 90.

2. "Hamlet and His Problems ", in The Sacred Wood, Essays on Poetry and Criticism, 1922.

3. A Midsummer Night's Dream, 3.1.25.

4. Paul Fussell Jr., Theory of Prosody in Eighteenth Century England, New London, Connecticut College, 1953.

\section{RÉSUMÉS}

Évoquer Shakespeare poète, c'est pour une partie du public le désigner comme le porte-parole des muses, virtuose et alchimiste du langage, visionnaire, prophète, chantre des beautés de la nature, de l'héroïsme et de l'amour, mais aussi peintre lucide et pathétique des misères et de la cruauté des hommes. Bien que ce type de dithyrambe paraisse démodé, on s'y livre parfois, d'où des effusions lyriques, voire mystiques, par lesquelles le commentateur tente de s'élever jusqu'à l'objet de son admiration. Cependant à l'origine un poète est plus humblement un créateur, un fabricant, capable d'invention mais sur une base artisanale, et dans d'autres domaines que la poésie pure, en l'occurrence le théâtre. Ce savoir-faire peut s'accompagner de réflexion esthétique, implicite ou explicite. Les relations dialectiques entre l'art et la nature forment un des axes de cette réflexion, et les remarques métapoétiques insérées par l'auteur dans ses œuvres éclairent sa conception de l'art et de sa fonction.

To the public mind in general the mere mention of Shakespeare as poet amounts to marking him out as the Muses' mouthpiece, a virtuoso and alchemist of language, a visionary, a prophet, a bard singing the glories of Nature, love and heroism, as well as a disillusioned yet pathetic painter of human miseries and cruelties. Though this type of dithyrambic praise may seem oldfashioned it is still practised, producing lyrical or even mystical effusions by the means of which the commentator tries to climb to the very height where the object of his or her idolatry is enthroned. Yet originally, a poet is more modestly a creator of literary compositions, a maker, capable of invention indeed, but based on craftsmanship, and in other fields than poetry in the restricted sense, for instance in the theatre. This savoir-faire can be accompanied, either explicitly or implicitly, by aesthetic remarks. Some of these bear on the relationships between art and nature, which constitute one of the topics of this article. The observations dealing with 
poetry itself that the author dropped in his works throw some light on his conception of art and of its function.

\section{AUTEUR}

\section{HENRI SUHAMY}

Professeur émérite de classe exceptionnelle à l'Université de Paris X, a enseigné en Algérie, au Sénégal, au CNED Élève de l'ENS de Saint-Cloud, agrégé, docteur d'État (Le vers de Shakespeare), fondateur de la Société de stylistique anglaise, traducteur de Shakespeare et de Scott, il a publié une centaine d'articles sur divers sujets, et 25 livres sur Shakespeare, Scott, Donne, Henri viII, la versification, la stylistique, la poétique. Lauréat du jury Chateaubriand et de l'Académie française. 